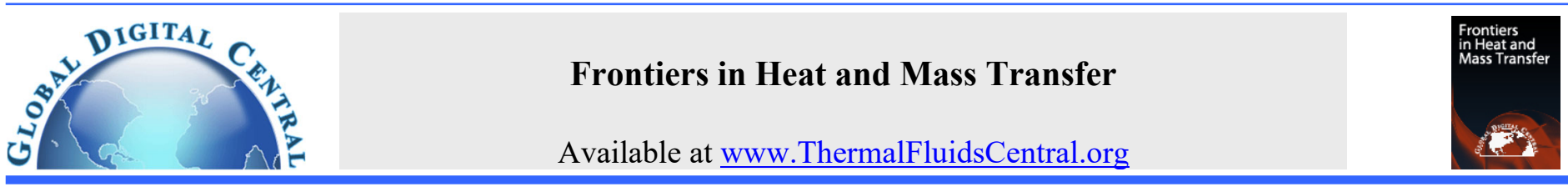

\title{
TRANSVERSAL FLOW AND HEAT TRANSFER OF TWO CYLINDERS WITH A FLAPPING REED BETWEEN THEM
}

\author{
Zhiyun Wang ${ }^{*}$, Ziqing Wang, Mo Yang \\ School of Energy and Power Engineering, University of Shanghai for Science and Technology, Shanghai, 200093, China
}

\begin{abstract}
This paper presents a two-dimensional fluid-structure interaction numerical simulation of fluid flow over two horizontal heat exchange cylinders affected by a flapping reed in a domain. The reed is a thin flexible sheet made of elastic material with one end fixed on the trailing edge of the upstream cylinder. The effects of the reed length and the cylinder spacing on the periodic oscillations of the reed, the flow field and the heat transfer of the downstream cylinder. The results show that the oscillation of the reed in this paper is a single-period oscillate model. Compared to the case of cylinder without any measurement of heat transfer enhancement (clean cylinder), the heat transfer performance of the cylinder with the reed could be enhanced under certain conditions. For the case of the cylinder spacing $\mathrm{S}^{*}=1.5$, as the length of the reed increases, the heat transfer of the downstream cylinder increases by up to $14 \%$ compared to the clean cylinder, while the domain resistance coefficient is almost unchanged. For the cases of cylinder spacing $\mathrm{S}^{*}=2.0$, as the length of the reed increases, the heat transfer of the downstream cylinder gradually decreases, but the domain resistance coefficient gradually increases to $12 \%$.
\end{abstract}

Keywords: fluid-structure interaction, heat and mass transfer, transversal flow, cylinders, numerical simulation

\section{INTRODUCTION}

In recent years, obtaining higher heat transfer efficiency through enhanced heat transfer technology has become a hot issue in heat exchanger. According to Bergles' (1999) classification method of heat transfer enhancement by convection, it can be roughly divided into the passive enhancement technology and the active enhancement technology. The active type must rely on external power, such as mechanical force or electromagnetic force, while the passive type does not need to use other forms of power besides the power required to transport the fluid medium. Therefore, compared with the active type, the passive type reduces cost consumption and enhances equipment reliability (Beskok et al., 2012; Pourgholam et al., 2015).

Heat transfer augmentations through the use of the passive type that enhance flow mixing and reduce mechanical loss have been the subject of much research effort. According to their movability, the passive enhancement technology can be divided into the fixed type and the flapping type. In the process of enhancing heat transfer, the flapping type produces deformation and displacement under the action of fluid. In various studies (Fiebig et al.,1993; Facchinetti et al., 2004; Yoo et al., 2002; Song and Wang, 2013), scholars had conducted in-depth studies on the enhancement of heat transfer performance of the fixed type under different shapes and tube spacing. About the flapping type (Li et al., 2018; Gallegos et al., 2017; Lee et al., 2017; Lee et al., 2018; Chen et al., 2020; Khanafer et al., 2010), researchers have made great efforts. They studied the influence of different shapes, different placement forms, and different physical parameters of the flapping reed on the heat transfer performance of the channel. Compared with the fixed type, the flapping type heat transfer performance is better, and the channel pressure loss is smaller. Instead of modifying the channel surface, flapping vortex generator made of elastic material can be placed in the existing channel directly. Therefore, the fluid-structure coupling passive flapping reed enhanced heat transfer has become a hot topic in many engineering fields (Facchinetti et al., 2004).

In summary, most of the previous studies focused on the influence of the flapping reed on the heat transfer of the channel, but did not study its' influence on the heat transfer of the heat exchanger tubes. Therefore, this article is based on the enhanced heat transfer of the flapping reed. Take the variation of reed length and the change of the cylinder spacing as the research focus. By means of two-dimensional fluid-structure interaction (FSI) numerical simulation, research on the heat transfer enhancement and the fluid flow characteristics.

\section{PROBLEM STATEMENT AND MATHEMATICAL MODEL}

\subsection{Physical Model}

The two-dimensional physical model is shown schematically in Fig. 1. The fluid enters the domain at the left boundary with a uniform velocity $U$ and low temperature $T_{i n}$ and then flow across two cylinders with higher temperature $T_{\text {wall }}$. There would be the heat exchange between the fluid and the cylinders due to the temperature difference in the domain. The diameter of the cylinder is $D$ and the length of the domain $C_{L}=12 D$. With the set of the domain height $C_{H}=4.1 D$ and the upper and lower boundaries walls are set to be symmetrical conditions. The setting is intentionally non-symmetric to prevent the dependence of the onset of any possible oscillation on the precision of the computation. The influence of upper and lower boundary to the flow and heat transfer can be ignored in this model (Khan et al., 2006). A flexible sheet is installed as the reed with one end fixed on the trailing edge of the upstream cylinders. The length of reed is $L$. The distance between the two cylinders is $S$. The right boundary is set to a constant pressure and 
far enough from the cylinders. The surfaces of the two cylinders are no slip boundary condition. The surface of the reed is adiabatic and the thickness of it is $H$ which is used only for the calculation of the solid inner stress.

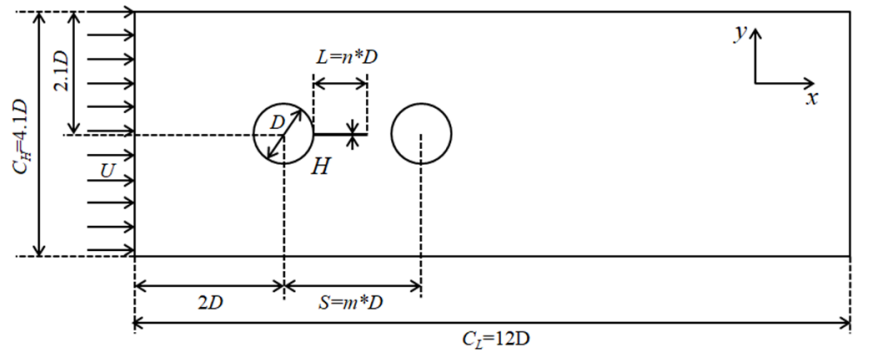

Fig. 1 Physical model

\subsection{Mathematical Model}

It is assumed in this investigation that the flow is two-dimensional, steady and incompressible. The FSI model calculation region contains the fluid domain and the solid domain. An arbitrary LagrangianEulerian formulation was adopted to describe the fluid motion. Furthermore, the governing equations for the fluid domain are the continuity and Navier-Stokes equations. Thus, the Navier-Stokes equations are described in tensor form as:

$\rho_{f} \frac{\partial u_{i}}{\partial t}+\rho_{f} u_{i, j}\left(u_{j}-\hat{u}_{j}\right)=\sigma_{i j, j}+\rho_{f} f_{i}$

$u_{i, i}=0$

Where $\rho_{f}$ is the fluid density, $u_{i}$ is the velocity tensor, $t$ is the time, $\hat{u}_{j}$ is the moving velocity of the solid region and indices indicate vector components and differentiation in index (notation), $\sigma_{i j, j}$ is the stress tensor and $f_{i}$ is the body force per unit mass. For incompressible fluids, the principle of conservation of thermal energy is expressed by:

$\rho_{f} C_{p}\left(\frac{\partial T}{\partial t}+u_{i} T_{, i}\right)=\left(\lambda_{f} T_{, i}\right)_{, i}$

Where $T$ is the temperature, $\lambda_{f}$ is the thermal conductivity of the fluid, and $C_{p}$ is the specific heat at constant pressure. In addition, the governing equation for the solid domain of the FSI model can be described by the following equation:

$\rho_{s} \ddot{d}_{i}=\bar{\sigma}_{i j, j}+\rho_{s} \bar{f}_{i}$

Where $\rho_{s}$ is the flapping reed density, $\bar{\sigma}_{i j, j}$ is the solid Cauchy stress tensor, $\bar{f}_{i}$ is the externally applied body force vector at time $t$ and $\ddot{d}_{i}$ represents the acceleration of the solid domain.

While the inlet and outlet boundary conditions implemented in this investigation are summarized as follows:

$x=0: u=U, \mathrm{v}=0, \mathrm{~T}=\mathrm{T}_{i n}$

$x=L: \frac{\partial u}{\partial x}=\frac{\partial v}{\partial x}=\frac{\partial T}{\partial x}=0$

About the top and bottom thermal boundaries and the thermal boundary conditions along the flapping reed are given by:

$y=0, \mathrm{C}_{H}$ and $0 \leq \mathrm{x} \leq \mathrm{C}_{L}: u=v=\frac{\partial T}{\partial y}=0$

The thermal boundaries on the surface of both cylinders are as follows:

$T=T_{\text {wall }}$

The final set of boundary conditions is the FSI interfaces such that the conditions of displacement compatibility and traction equilibrium along the structure-fluid interfaces must be satisfied as follows respectively:

$d_{f}=d_{s}$

$\sigma_{f}=\sigma_{s}$
Where $d_{f}$ and $d_{s}$ are the displacements, $\sigma_{f}$ and $\sigma_{s}$ are the tractions of the fluid and the solid.

\subsection{Dimensionless Parameter Definition}

The problem is then described by 7 dimensionless parameters: $S^{*}=\frac{S}{D}, L^{*}=\frac{L}{D}, H^{*}=\frac{H}{D}$,

$\rho^{*}=\frac{\rho_{s}}{\rho_{f}}, E^{*}=\frac{E}{\rho_{f} U^{2}}, v_{s}=0.4$,

$\operatorname{Re}=\frac{U D}{v_{f}}$

Where the superscript ${ }^{*}$ represents non-dimensional parameters. $H^{*}$ represents the non-dimensional thickness of the reed, which is a constant and the value is 0.2 . The elasticity of the solid material is characterized by the Poisson ratio $v_{s}$ and $E^{*}$, which are fixed at 0.4 and 2.5 respectively in this article. The Reynolds number used in this study is fixed at 200 for all cases. The heat transfer performance is evaluated by $\mathrm{Nu}$ number, which is defined as follows:

$$
\begin{aligned}
& N u_{\text {ave }}=\frac{\bar{h} D}{\lambda} \\
& N u_{\alpha}=\frac{h_{\alpha} D}{\lambda}
\end{aligned}
$$

Where $N u_{\text {ave }}$ and $N u_{\alpha}$ are the average heat transfer and local transfer performance of the downstream cylinder. In the expression, $\bar{h}$ and $h_{\alpha}$ are the average convection heat transfer coefficient and local convection heat transfer coefficient of the downstream cylinder. They are summarized as follows:

$$
\begin{aligned}
& \bar{h}=\frac{1}{2 \pi} \int_{0}^{2 \pi} h \alpha d \alpha \\
& h_{\alpha}=\frac{-\lambda \frac{\partial t}{\partial n}}{T_{\text {wall }}-T_{\text {in }}}
\end{aligned}
$$

Where $\alpha$ is the angle between the calculation point on the cylinder and the direction of the incoming flow. The resistance coefficient is used to evaluate the pressure drop of the fluid in all condition, which is as follows:

$$
C_{D}=\frac{2 \Delta p}{\rho_{f} U^{2} D}
$$

In this expression, $\Delta p$ is the domain pressure drop. The dimensionless amplitude of the free end of the flapping reed is shown as:

$A_{y}^{*}=\frac{A_{y}}{D}$

Where $A_{y}$ is the maximum amplitude of the free end of the flapping reed in the direction perpendicular to the incoming fluid.

\subsection{Numerical Method}

Simulation method used in this article is finite element formulation based on the Galerkin method, which was employed to solve the governing equations of a FSI model. According to the boundary conditions in Eqs. (4) and (8), the finite element method was used to discretize the continuity and momentum formulas. These equations are weighted with the virtual quantities of pressure and velocities. A variable grid-size system was employed to capture the rapid changes in the dependent variables especially near the wall where the major gradients occur inside the boundary layer. In addition, the NewtonRaphson method was used to solve the discretized equations for the fluid and solid domain. The time step size of $0.005 \mathrm{~s}$ was used until periodic convergence solution is achieved. 


\section{VERIFICATION NUMERICAL METHOD AND GRID INDEPENDENCE}

\subsection{Numerical Simulation Method Verification}

The commercial software COMSOL Multiphysics is employed to simulate the FSI process. In order to verify the numerical method, the problems shown in Fig.2 are simulated and result of $A_{y}^{*}$ and $C_{D}$ are compared with those reported by Turek et al. (2006) and Tian et al. (2014). In Fig.2, the fluid enters the domain at the left boundary with a parabolic velocity $U$ which average is $\bar{U}$. The upper and lower boundaries are the walls. The dimensionless length of the physical model is shown in the Fig. 2.

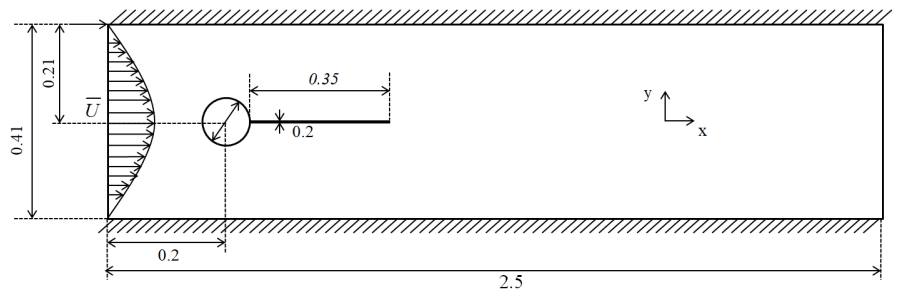

Fig. 2 Physical model of verify numerical method

The detail of comparison is listed in Table 1. It can be seen that the results obtained by the numerical method employed in this paper are consistent with the previous researches, which indicate that the numerical simulation method used in this paper is feasible.

Table 1 Verification of $A^{*}{ }_{v}$ and $C_{D}$ results

\begin{tabular}{|l|l|l|l|}
\hline Physical parameters & Comparative case & $A_{y}^{*}$ & $C_{D}$ \\
\hline \multirow{2}{*}{$\rho^{*}=10 \mathrm{Re}=100$} & Turek & 0.83 & 4.13 \\
\cline { 2 - 4 }$E^{*}=1.4 \times 10^{3}$ & Tian & 0.78 & 4.11 \\
\cline { 2 - 4 } & Present result & 0.7836 & 4.1205 \\
\hline \multirow{2}{*}{$\rho^{*}=1 \mathrm{Re}=200$} & Turek & 0.36 & 2.30 \\
\cline { 2 - 4 }$E^{*}=1.4 \times 10^{3}$ & Tian & 0.32 & 2.16 \\
\cline { 2 - 4 } & Present result & 0.3189 & 2.1723 \\
\hline
\end{tabular}

\subsection{Grid independence verification}

The structured meshing is used for the physical model and the grid near the cylinders is densified. Different grid size of $38000,46800,65050$, $81050,102050,115000,122000,136050$ and 140600 are employed respectively for the case of $S^{*}=2.0$ and $L^{*}=0.7$. Figure 3 shows a diagram of $A^{*} y$ and $N u_{\text {ave }}$ under different grids size. Grid independence is achieved within $0.06 \%$ in $A^{*} y$ number and $0.18 \%$ in $N u_{a v e}$ with grid size of 122,000 which will be used for the follow-up calculations.

\section{RESULTS AND DISCUSSION}

$S^{*}=1.5$ and 2.0 are considered in this paper, since they represent the typical spacing adapt in industry that may cause stagnation vortexes between cylinders and would lead to heat transfer weakness (Marsters, 1972). The set of flapping reed between two cylinders may be adverse to the formation of stable stagnation vortexes.

\subsection{Effects of the Length of Reed Under $S^{*}=1.5$}

When $S^{*}=1.5$ and the length of the reed $L^{*}=0.10,0.25,0.30,0.35$ and 0.40 is investigated. Under this condition, the reed is the single-period flapping model (Connell et al. 2007). Figure 4 shows the $A_{y}^{*}$ change with time under the limit condition of $L^{*}=0.10$ and 0.40 and the Fourier analysis results. There is an obvious peak at a certain frequency, while it can be inferred that the flapping model of reed is a self-sustaining periodic oscillation.

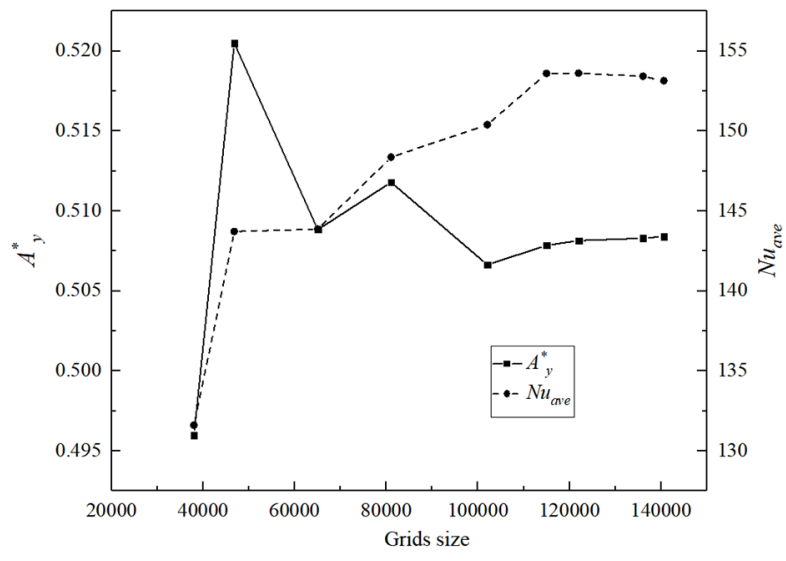

Fig. $3 A^{*}{ }_{y}$ and $N u_{a v e}$ under different grids size

The percentage change rate of downstream cylinder $N u_{a v e}$ and the domain $C_{D}$ under different reed length are compared with those of the clean cylinder and shown in Fig. 5. When the $L^{*}$ is increased from 0 to $0.25, N u_{\text {ave }}$ and $C_{D}$ hardly change. As $L^{*}$ further increases to 0.40 , the percentage change rate of $N u_{a v e}$ is reaches up to $12 \%$. Simultaneously, $C_{D}$ changes little.

Figure 4 shows amplitude of the free end of the reed $A_{y}$ in the direction perpendicular to the mainstream is stable after $\mathrm{t}=2.5 \mathrm{~s}$ and $2.9 \mathrm{~s}$, respectively and the oscillation of the reed is single periodic. Therefore, it is possible to simplify the analysis of $N u_{\alpha}$ on the downstream cylinder, and only analyze the upper half of the cylinder. All cases in this article, after 7 periods of stable oscillation of the reed, the heat transfer becomes stable, and the time average $N u_{\alpha}$ is calculated from 8 to 12 oscillation periods. $\alpha\left(0^{\circ} \leq \alpha \leq 180^{\circ}\right)$ is the angle between the direction of the incoming flow and the point on the surface of the cylinder, as shown in Fig. $6 . N u_{\alpha}$ at $\alpha=0^{\circ}$ is lower than other place. It is because that the distance between the cylinders is small which causes a flow stagnation point at the leading edge point of the downstream cylinder. As $\alpha$ increase to $50^{\circ}, N u_{\alpha}$ gradually increases, and then keeps a relative higher value until $\alpha \leq 80^{\circ}$. As the results of the twin vortices between the cylinders enhance fluid turbulence and heat transfer. At $50^{\circ} \leq \alpha \leq 125^{\circ}$, the heat transfer weakens due to the boundary layer gradually thickens. Along with $\alpha$ increase up to $180^{\circ}$, fluid departs from the surface of the cylinder which enhanced heat transfer. Results in local $N u_{\alpha}$ rebounded.

To quantify the difference, this paper selects the representative conditions when $L^{*}=0.25$ and 0.40 . Comparing the condition when $L^{*}=0$ and 0.25 , it is obviously that $N u_{\alpha}$ has a certain increase in the range of $0^{\circ} \leq \alpha \leq 7^{\circ}$, result of the flapping of the reed destroys the front stagnation point of the downstream cylinder. While in the range of $7^{\circ} \leq \alpha \leq 180^{\circ}$ the oscillating motion has almost no effect on the $N u_{\alpha}$, due to the shorter length of the reed which has little effect on the vortex between the cylinders and has almost no effect on the $N u_{\alpha}$. Comparing of $L^{*}=0$ and 0.40 shown in Fig.6, $N u_{\alpha}$ with the reed is obviously higher than $N u_{\alpha}$ of the clean cylinder at $0^{\circ} \leq \alpha \leq 18^{\circ}$ and $113^{\circ} \leq \alpha \leq 180^{\circ}$. Within the range of $18^{\circ} \leq \alpha \leq 113^{\circ}$, there is not much difference.

The reed flapping for one period under the conditions of $L^{*}=0$ and 0.40 the temperature gradient map are shown in Fig. 7. At $t=4.04 \mathrm{~s}$, $4.090 \mathrm{~s}$ and $4.15 \mathrm{~s}$, during $0^{\circ} \leq \alpha \leq 7^{\circ}$ due to the small distance between the cylinders, there is a stable twin vortex in the flow between the cylinders, while the left end of the downstream cylinder is the stagnation point. The flapping reed thins or destroys the thermal boundary layer on the left end of the downstream cylinder, which also increases the chaos of the fluid between the cylinders, increases the temperature gradient, and 
enhances the heat transfer performance. Fig. 8 shows the vorticity diagrams under the same condition. Due to the flapping of the reed, the vorticity at A and B marked in Fig. 8 is stronger than the vortex at same position of the clean cylinder. As the result of the increased vorticity, the vortices on the upper and lower sides of the downstream cylinder are squeezed and become smaller. This phenomenon brings the flow dead zone reduction and the mixing of the fluid of mainstream and the fluid at the downstream cylinder surface. Ultimately, heat transfer is enhanced.

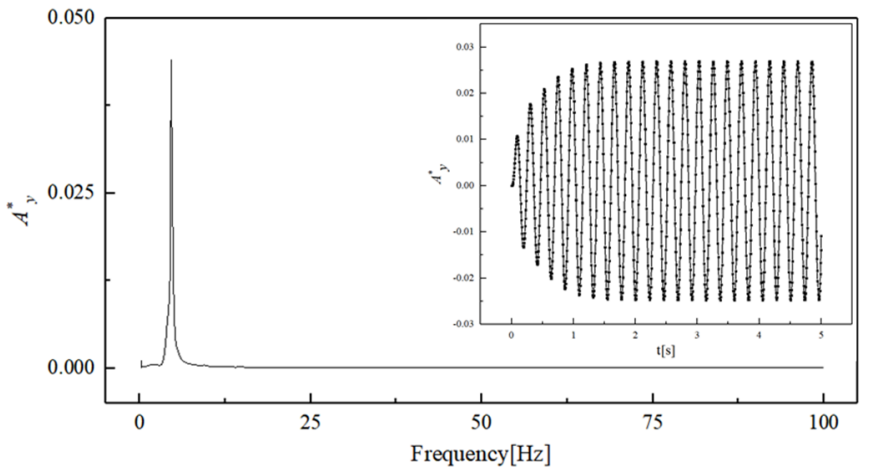

(a) $L^{*}=0.10$

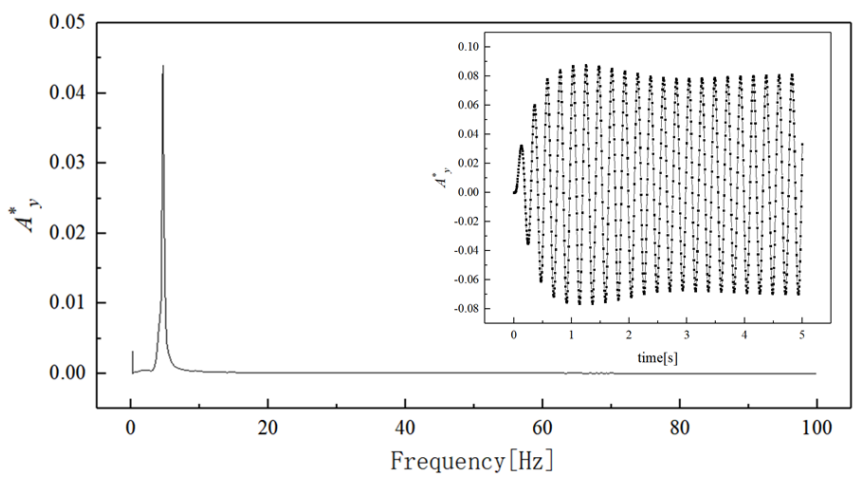

(b) $L^{*}=0.40$

Fig. $4 A_{y}^{*}$ change with time and the Fourier analysis results

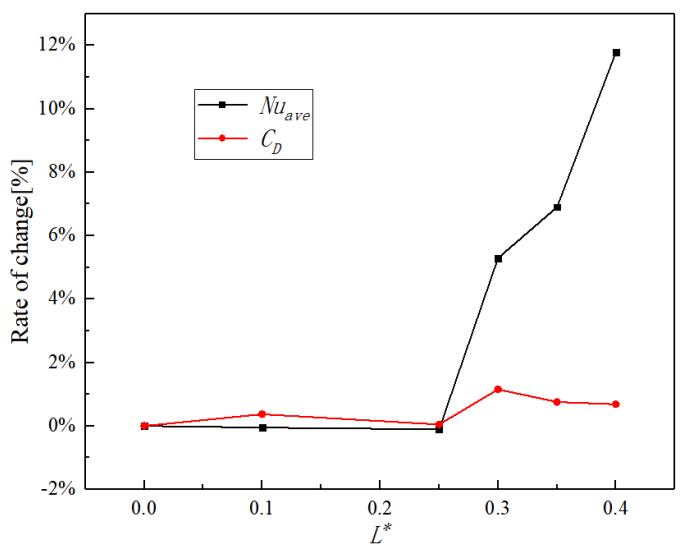

Fig. 5 Under $S^{*}=1.5$ trend of $N u_{\text {ave }}$ and $C_{D}$ when the $L^{*}$ changes

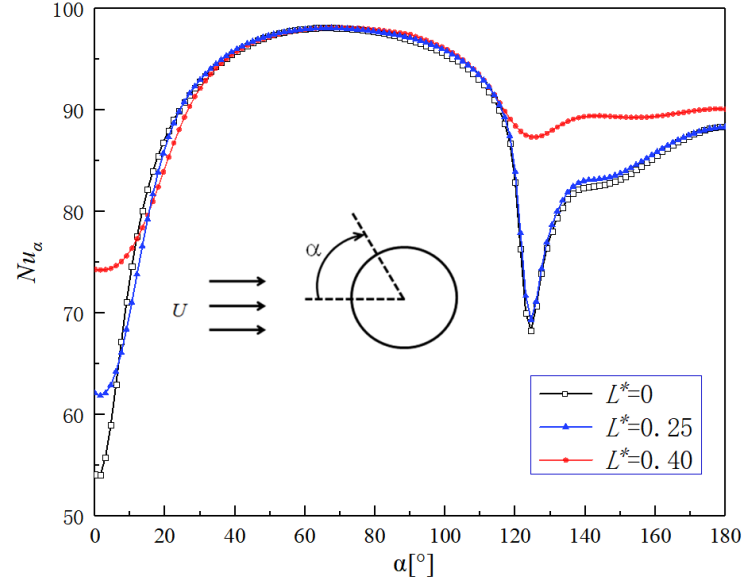

Fig. $6 L^{*}=0,0.25$ and 0.40 Variation trend of $N u_{a}$ with $\alpha$

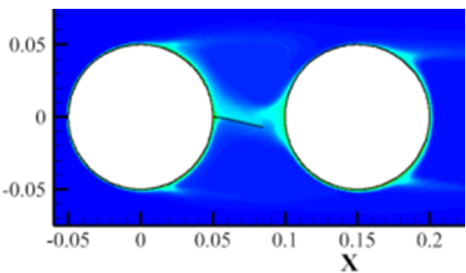

(a) $\mathrm{t}=4.04 \mathrm{~s} L^{*}=0.40$

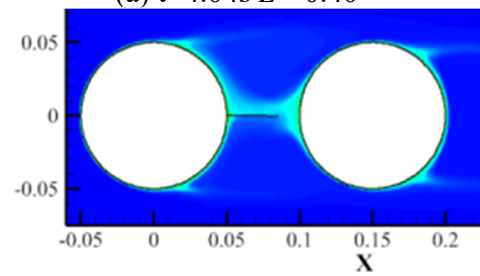

(c) $\mathrm{t}=4.09 \mathrm{~s} L^{*}=0.40$

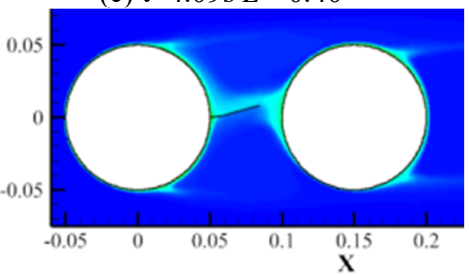

(e) $\mathrm{t}=4.15 \mathrm{~s} L^{*}=0.40$

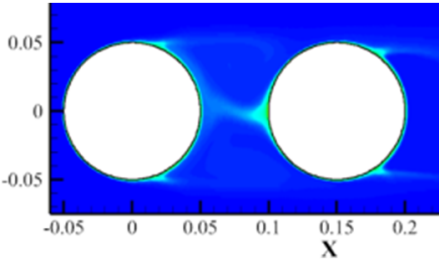

(b) $\mathrm{t}=4.04 \mathrm{~s} L^{*}=0$

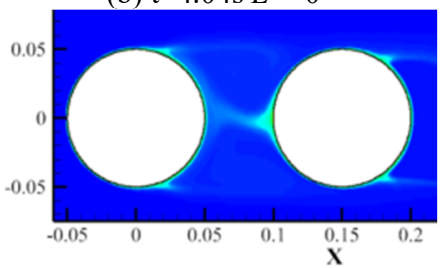

(d) $\mathrm{t}=4.09 \mathrm{~s} L^{*}=0$

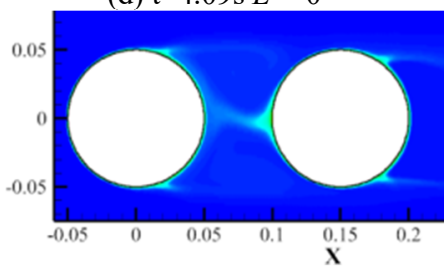

(f) $\mathrm{t}=4.15 \mathrm{~s} L^{*}=0$

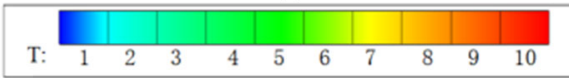

Fig. $7 L^{*}=0$ and 0.40 temperature gradient at different times

\subsection{Effects of the Length of Reed Under $S^{*}=2.0$}

Under the condition of $S^{*}=2.0$, along with the length of the reed $\mathrm{L} *$ changes, $N u_{\text {ave }}$ and $C_{D}$ have a completely different trend from previous case. The analysis method is the same as $S^{*}=1.5$. As shown in Fig. 9, the reed flapping, it also can be inferred that the flapping model of reed is a self-sustaining periodic oscillation. Fig. 10 shows under $S^{*}=2.0$ change the percentage change rate of $N u_{\text {ave }}$ and $C_{D}$ when the $L^{*}$ changes, obviously contrary to the previous trend. Fig. 10 shows that the reed length increases from 0 to $0.25 D$, the percentage change rate of $N u_{\text {ave }}$ increases by $10.58 \%$, and the $C_{D}$ hardly changes. As the length of the reed further increases, the $N u_{a v e}$ decreases until no difference from the clean cylinder. Simultaneously, $C_{D}$ significantly increases to about $12 \%$. 

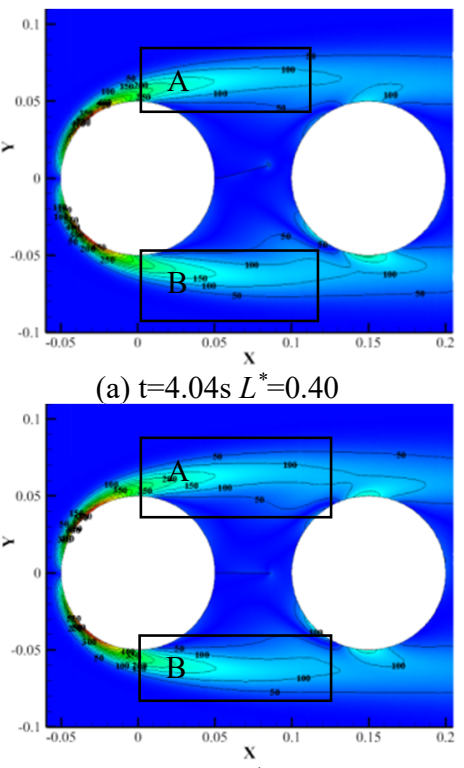

(c) $\mathrm{t}=4.09 \mathrm{~s} L^{*}=0.40$

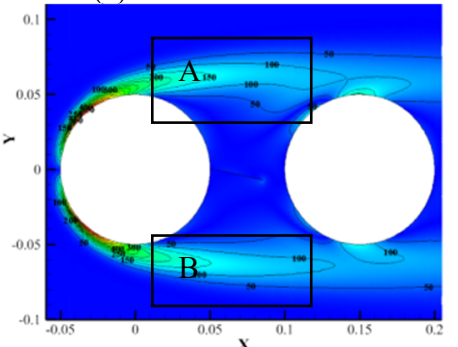

(e) $\mathrm{t}=4.15 \mathrm{~s} L^{*}=0.40$ (a) $\mathrm{t}=4.04 \mathrm{~s} \stackrel{\mathrm{x}}{L}^{*}=0.40$

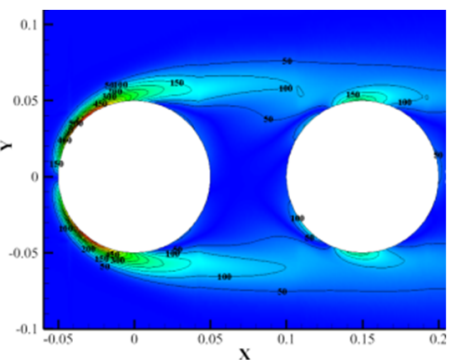

(b) $\mathrm{t}=4.04 \mathrm{~s} L^{*}=0$

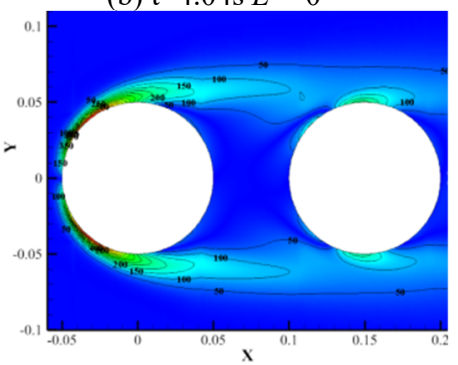

(d) $\mathrm{t}=4.09 \mathrm{~s} L^{*}=0$

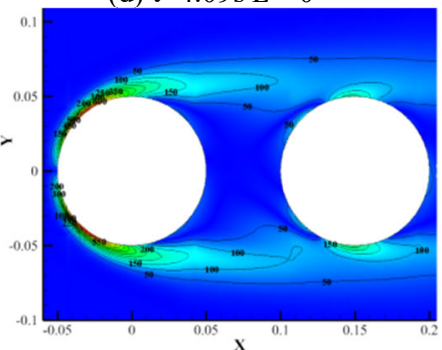

(f) $\mathrm{t}=4.15 \mathrm{~s} L^{*}=0$

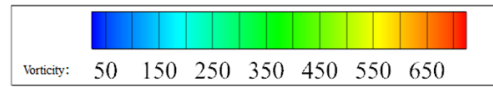

Fig. $8 L^{*}=0$ and 0.40 Vorticity at different times

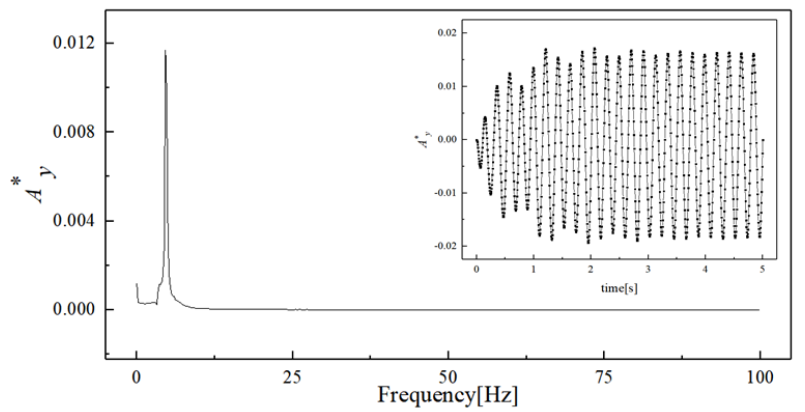

(a) $L^{*}=0.25$

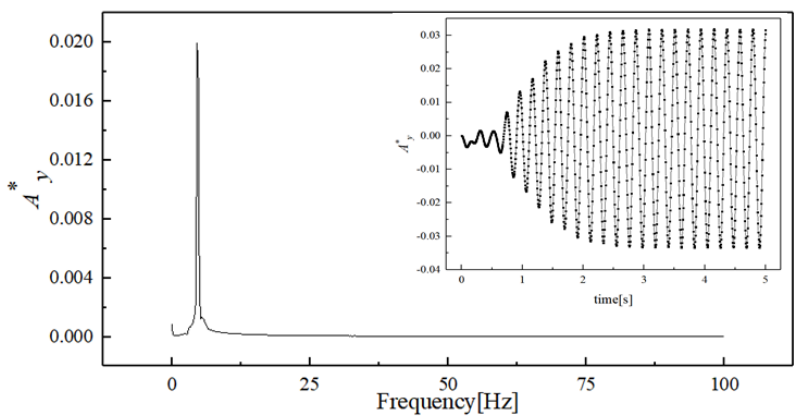

(b) $L^{*}=0.70$

Fig. $9 L^{*}=0.25$ and $0.70 A^{*} y$ change with time and the Fourier analysis results
Under $S^{*}=2.0$, the trend of $N u_{\alpha}$ while $\alpha$ changed is shown in Fig. 11. It is obvious that when $L^{*}=0.25, N u_{\alpha}$ at any $\alpha$ is greater than $L^{*}=0$. The Fig. 11 describes the flapping of the reed for a period the temperature gradient at different times. It can be seen from Fig.11, the flow between the tubes without the reed is basically in the symmetrical. There are obvious vortices between the cylinders, and symmetrical twin vortices appear. But when $L^{*}=0.25$, the disturbance between the cylinders increases, which strengthens the mixing of the fluid between the cylinders and the main fluid. Comparing Fig. 13 the temperature gradient graph at $L^{*}=0.70$ with Fig.12, after the length of the reed increases, the flapping amplitude increases. And because the existence of the reed hinders the mixing of the upper and lower parts fluid of the reed, the range of the dead zone increases, and the heat transfer of the downstream circular pipe is inhibited.

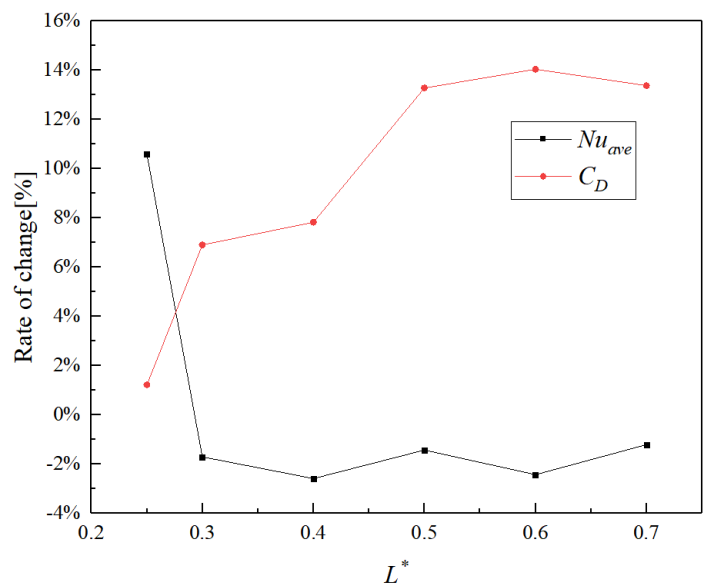

Fig. 10 Under $S^{*}=2.0$ trend of $N u_{\text {ave }}$ and $C_{D}$ when the $L^{*}$ changes

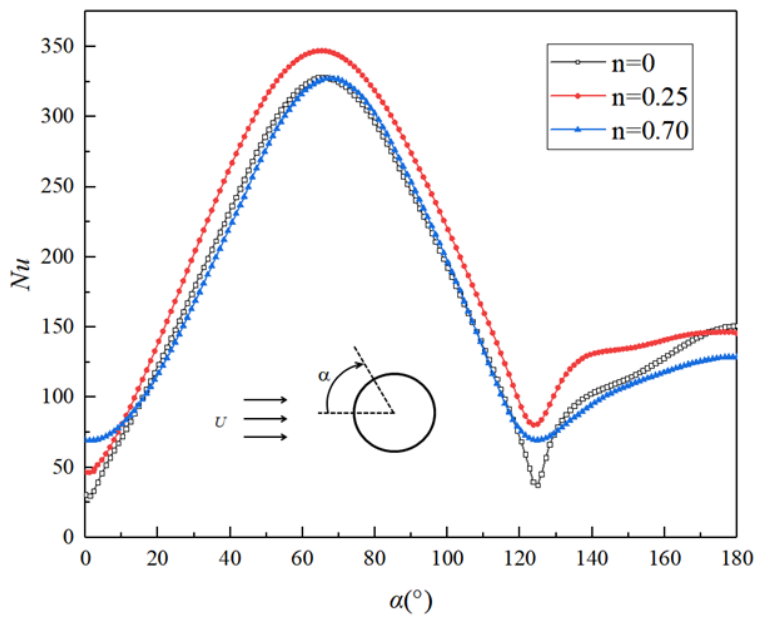

Fig. $11 L^{*}=0,0.25$ and 0.70 Variation trend of $N u_{\alpha}$ with $\alpha$

\section{CONCLUSION}

In this paper, a two-dimensional numerical simulation study of the effect of a flapping reed installed between the cylinders on the heat transfer and fluid flow effect is carried out. Mainly analyze the influence of the periodic flapping of the reed with different cylinder spacing and different length on the flow field and the heat transfer of the downstream cylinder. The result shows:

1) When the cylinder spacing $S^{*}=1.5$ is small, as the length of the reed increases from $L^{*}=0.10$ to 0.40 , the heat transfer performance of the downstream cylinder is continues to increase. At this time, due to the small distance between the cylinders, there is a stable twin vortex between them, and the left side of the downstream cylinder is the front 
stagnation point. After reed is added, the thermal boundary layer on the left side of the cylinder is destroyed, the degree of confusion of the fluid near the surface is increased and the heat transfer performance is enhanced.

2) In the case of the cylinder spacing $S^{*}=2.0$ as the length of the reed increases from 0.10 to 0.70 , the heat transfer performance of downstream cylinder continues to decrease until similar to clean cylinder. When the length of the reed is small, the flapping of the flexible body increases the disturbance between the cylinders. However, with the length increases, the reed hinders the flow of the upper and lower parts of itself. Eventually lead to the dead zone area is increased, and the heat transfer of the downstream cylinder is suppressed.

3) Due to the existence of flapping reed, the stagnation point at the front point the downstream cylinder is destroyed, and the heat transfer performance is significantly increased.

4) When the cylinder spacing $S^{*}=2.0$, as the length of the reed increases, the domain $C_{D}$ gradually increase. Due to existence of the reed, suppress fluid mixing between cylinders.

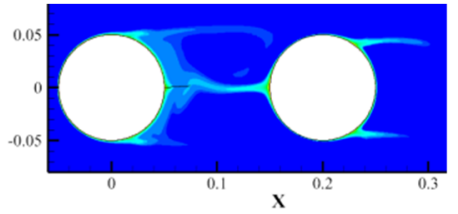

(a) $\mathrm{t}=4.84 \mathrm{~s}$

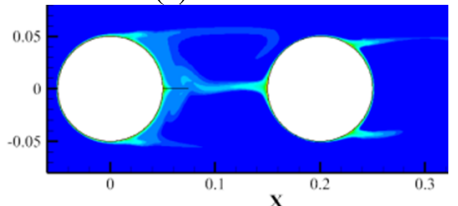

(c) $\mathrm{t}=4.89 \mathrm{~s}$

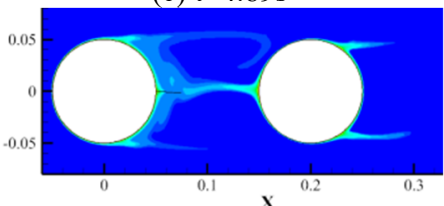

(e) $\mathrm{t}=4.94 \mathrm{~s}$

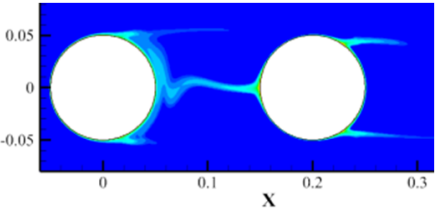

(b) $\mathrm{t}=4.84 \mathrm{~s}$

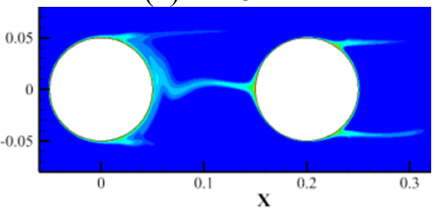

(d) $\mathrm{t}=4.84 \mathrm{~s}$

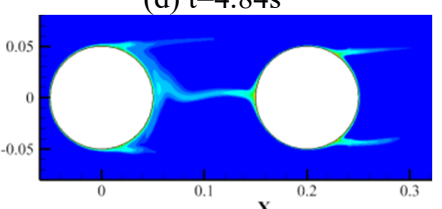

(f) $\mathrm{t}=4.84 \mathrm{~s}$

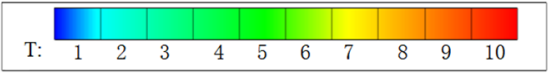

Fig.12 $L^{*}=0$ and 0.25 temperature gradient at different times

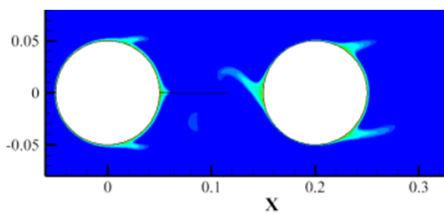

(a) $\mathrm{t}=5.25 \mathrm{~s}$

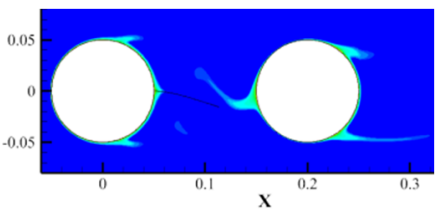

(b) $\mathrm{t}=5.85 \mathrm{~s}$

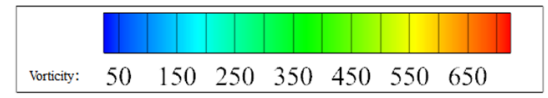

Fig. $13 L^{*}=0.70$ temperature gradient at different times

\section{NOMENCLATURE}

$A$

$C_{p}$

$C_{L-}$

$C_{H}$

D

d

E

H amplitude (m)

specific heat $(\mathrm{J} /(\mathrm{kg} \cdot \mathrm{K}))$

domain length $(\mathrm{m})$

domain height $(\mathrm{m})$

diameter of the cylinder $(\mathrm{m})$

displacements(m)

Young's modulus $(\mathrm{Pa})$

reed thickness $(\mathrm{m})$ $h \quad$ convection heat transfer coefficient $\left(\mathrm{W} /\left(\mathrm{m}^{2} \cdot \mathrm{K}\right)\right)$

$L \quad$ reed length (m)

$S \quad$ cylinders spacing (m)

$T \quad$ temperature (K)

$t \quad$ time (s)

$U \quad$ fluid velocity $(\mathrm{m} / \mathrm{s})$

Greek Symbols

$\rho \quad$ density $\left(\mathrm{kg} / \mathrm{m}^{3}\right)$

$\lambda \quad$ thermal conductivity $(\mathrm{W} /(\mathrm{m} \cdot \mathrm{K}))$

Superscripts

* dimensionless parameters

Subscripts

$f \quad$ fluid

$s \quad$ solid

\section{REFERENCES}

Bergles, E., 2007, "The imperative to enhance heat transfer," Springer Netherlands, 13-29.

https://doi.org/10.1007/978-94-015-9159-1 2

Beskok, A., Raisee, M., Celik, B., Yagiz, B., and Cheraghi, M., 2012,

"Heat transfer enhancement in a straight channel via a rotationally oscillating adiabatic cylinder," International Journal of Thermal Sciences, 58, 61-69.

https://doi.org/10.1016/j.ijthermalsci.2012.03.012

Connell, B. S. H., and Yue, D. K. P., 2007, "Flapping dynamics of a flag in a uniform stream," Journal of Fluid Mechanics, 581, 33-67. https://doi.org/10.1017/S0022112007005307

Chen, Y., Yang, J., Liu, Y., and Sung, H. J., 2020, "Heat transfer enhancement in a poiseuille channel flow by using multiple wallmounted flexible flags," International Journal of Heat and Mass Transfer, 163, 120447.

https://doi.org/10.1016/j.ijheatmasstransfer.2020.120447

Fiebig, M., Valencia, A., and Mitra, N. K., 1993, "Local Heat Transfer and Flow Losses in Fin-Tube Heat Exchangers with Vortex Generators: A Comparison of Round and Flat Tubes," Experimental Thermal \& Fluid Science, 8(2), 35-45.

https://doi.org/10.1016/0894-1777(94)90071-X

Facchinetti, M. L., Langre, E. D., and Biolley, F., 2004, "Coupling of structure and wake oscillators in vortex-induced vibrations," Journal of Fluids and Structures, 19(2), 123-40.

https://doi.org/10.1016/j.jfluidstructs.2003.12.004

Gallegos, Ralph, K. B., and Sharma, R. N., 2017, "Flags as vortex generators for heat transfer enhancement: Gaps and challenges," Renewable and Sustainable Energy Reviews, 76, 950-962.

https://doi.org/10.1016/j.rser.2017.03.115

Khan, W. A., Culham, J. R., and Yovanovich, M. M., 2006, "Convection heat transfer from tube banks in crossflow: Analytical approach," International Journal of Heat and Mass Transfer, 49(25), 4831-4838.

https://doi.org/10.1016/j.ijheatmasstransfer.2006.05.042

Khanafer, K., ALAMIRI, A., and POP, I., 2010, "Fluid-structure interaction analysis of flow and heat transfer characteristics around a flexible microcantilever in a fluidic cell," International Journal of Heat and Mass Transfer, 53(9-10), 1646-1653.

https://doi.org/10.1016/j.ijheatmasstransfer.2010.01.029

Lee, J. B., Park, S. G., Kim, B., Ryu, J., and Sung, H. J., 2017, "Heat transfer enhancement by flexible flags clamped vertically in a Poiseuille channel flow," International Journal of Heat and Mass Transfer, 107, 391-402.

https://doi.org/10.1016/j.ijheatmasstransfer.2016.11.057 
Lee, J. B., Park, S. G., and Sung, H. J., 2018, "Heat transfer enhancement by asymmetrically clamped flexible flags in a channel flow," International Journal of Heat and Mass Transfer, 116, 10031015. https://doi.org/10.1016/j.ijheatmasstransfer.2017.09.094

Li, Z., Xu, X. C., Li, K. J., Chen, Y. Y., Huang, G. L., Chen, C. L., and Chen, C. H., 2018, "A flapping vortex generator for heat transfer enhancement in a rectangular airside fin," International Journal of Heat and Mass Transfer, 118, 1340-1356.

https://doi.org/10.1016/j.ijheatmasstransfer.2017.11.067

Marsters, G. F., 1972, "Arrays of heated horizontal cylinders in natural convection," International Journal of Heat \& Mass Transfer, 15(5), 921-933.

https://doi.org/10.1016/0017-9310(72)90231-1

Pourgholam, M., Izadpanah, E., Motamedi, R., and Habibi, S. E., 2015. "Convective heat transfer enhancement in a parallel plate channel by means of rotating or oscillating blade in the angular direction," Applied Thermal Engineering, 78(78), 248-257.

https://doi.org/10.1016/j.applthermaleng.2014.12.057

Sohankar, A., 2007, "Heat transfer augmentation in a rectangular channel with a vee-shaped vortex generator," International Journal of Heat and Fluid Flow, 28(2), 306-317.

https://doi.org/10.1016/j.ijheatfluidflow.2006.03.002
Song, K. W., and Wang, L. B., 2013, "The Effectiveness Of Secondary Flow Produced by Vortex Generators Mounted on Both Surfaces of the Fin to Enhance Heat Transfer in a Flat Tube Bank Fin Heat Exchanger," Journal of Heat Transfer, 135(4), 041902. https://doi.org/10.1115/1.4023037

Turek, S., and Hron, J., 2006, "Proposal for Numerical Benchmarking of Fluid-Structure Interaction between an Elastic Object and Laminar Incompressible Flow," Springer Berlin Heidelberg, 371-385. https://doi.org/10.1007/3-540-34596-5_15

Tian, F. B., Dai, H., Luo, H., Doyle, J. F., and Rousseau, B., 2014, "Fluid-structure interaction involving large deformations: 3D simulations and applications to biological systems," Journal of Computational Physics, 258, 451-469. https://doi.org/10.1016/i.jcp.2013.10.047

Yoo, S. Y., Park, D. S., and Chung M. H., 2002, "Heat Transfer Enhancement For Fin-Tube Heat Exchanger Using Vortex Generators," Journal of Mechanical Science and Technology, 16(1), 109-115. https://doi.org/10.1007/BF03185161 